\title{
EVIDENCE FOR QUASI-PERIODIC X-RAY DIPS FROM AN ULTRALUMINOUS X-RAY SOURCE: IMPLICATIONS FOR THE BINARY MOTION
}

\author{
Dheeraj R. Pasham ${ }^{1}$ and Tod E. Strohmayer ${ }^{2}$ \\ ${ }^{1}$ Astronomy Department, University of Maryland, College Park, MD 20742, USA; dheeraj@astro.umd.edu \\ 2 Astrophysics Science Division, NASA's Goddard Space Flight Center, Greenbelt, MD 20771, USA; tod.strohmayer@nasa.gov \\ Received 2012 March 19; accepted 2012 December 7; published 2013 January 29
}

\begin{abstract}
We report results from long-term $(\approx 1240$ days $) \mathrm{X}$-ray $(0.3-8.0 \mathrm{keV})$ monitoring of the ultraluminous $\mathrm{X}$-ray source NGC 5408 X-1 with the Swift/X-Ray Telescope. Here we expand on earlier work by Strohmayer (2009) who used only a part of the present data set. Our primary results are: (1) the discovery of sharp, quasi-periodic, energyindependent dips in the X-ray intensity that recur on average every 243 days, (2) the detection of an energy dependent (variability amplitude decreases with increasing energy), quasi-sinusoidal X-ray modulation with a period of 112.6 \pm 4 days, the amplitude of which weakens during the second half of the light curve, and (3) spectral evidence for an increase in photoelectric absorption during the last continuous segment of the data. We interpret the X-ray modulations within the context of binary motion in analogy to that seen in high-inclination accreting X-ray binaries. If correct, this implies that NGC $5408 \mathrm{X}-1$ is in a binary with an orbital period of $243 \pm 23$ days, in contrast to the 115.5 day quasi-sinusoidal period previously reported by Strohmayer (2009). We discuss the overall X-ray modulation within the framework of accretion via Roche-lobe overflow of the donor star. In addition, if the X-ray modulation is caused by vertically structured obscuring material in the accretion disk, this would imply a high value for the inclination of the orbit. A comparison with estimates from accreting X-ray binaries suggests an inclination $\gtrsim 70^{\circ}$. We note that, in principle, a precessing accretion disk could also produce the observed X-ray modulations.
\end{abstract}

Key words: accretion, accretion disks - methods: data analysis - X-rays: binaries

\section{INTRODUCTION AND BACKGROUND}

Ultraluminous X-ray sources (ULXs) are bright, point-like, extragalactic sources with $\mathrm{X}$-ray luminosities in the range from a few $\times 10^{39} \mathrm{erg} \mathrm{s}^{-1}$ to as high as $10^{41} \mathrm{erg} \mathrm{s}^{-1}$ (e.g., Swartz et al. 2004 and references therein). The nature of the physical process producing such high X-ray output is not completely understood, but there is now strong evidence that some ULXs are accretionpowered systems containing a black hole. What remains more controversial is the mass of the accretor.

Current arguments suggest that these sources are either stellar-mass black holes (mass range: $3-50 \quad M_{\odot}$ ) accreting via a super-Eddington mechanism (e.g., Körding et al. 2002; King et al. 2001; Begelman 2002) or that they comprise an intermediate-mass black hole (mass range: a few $\left.\times(100-1000) M_{\odot}\right)$ accreting at sub-Eddington accretion rates (Colbert \& Mushotzky 1999). It is possible that the population of ULXs is an inhomogeneous sample with both types of sources present. A search for intermediate-mass black holes is important as they are often required as building blocks to explain the formation of the super-massive black holes (Volonteri et al. 2003; Davies et al. 2011) that reside at the centers of almost all massive galaxies (Magorrian et al. 1998). In this work we focus on the ULX NGC 5408 X-1, one of the promising candidates for an intermediate-mass black hole (Strohmayer \& Mushotzky 2009). Here we present evidence for the detection of quasi-periodic dips in its X-ray light curve that likely trace the orbital motion of the system.

There are now two detections of periodicities from long-term ( $\sim$ a few hundred days) X-ray monitoring of ULXs which may reflect the orbital motion of these systems: a 62-day modulation in M82 X-1 detected with RXTE (Kaaret \& Feng 2007) and a 115.5-day period in NGC 5408 X-1 obtained from Swift data (Strohmayer 2009, S09 hereafter). The main results of S09 are:
(1) the discovery of a quasi-sinusoidal modulation of the X-ray flux with a period of $115.5 \pm 4$ days and (2) that the modulation amplitude decreases with increasing energy. The present work utilizes additional data acquired by Swift over a much longer temporal baseline (more than twice as long as used by S09) and can be regarded as an extension of the work by S09.

The phenomenon of periodic orbital X-ray modulations within the context of galactic X-ray binaries has been well studied for over 30 years now (e.g., White \& Holt 1982; Mason 1986). The basic idea is that there is a distribution of obscuring material around the X-ray emitting region and as the X-ray source orbits the center of mass of the binary, our line of sight intercepts varying amounts of the intervening material resulting in the observed modulation (e.g., Parmar \& White 1988; Armitage \& Livio 1998). In addition, these X-ray variations are expected to recur with the orbital period of the binary. However, due to the turbulent nature of the accretion process and irregularities within the material surrounding the X-ray source, these modulations may not be strictly periodic (see, for example, Smale et al. 1988; Barnard et al. 2001; Kuulkers et al. 2012). Nevertheless, they provide an excellent means to track the orbital motion of the X-ray source.

Moreover, the nature of the obscuring medium, i.e., its distribution around the X-ray source as well as its density and ionization state, dictates the observed modulation profiles. In the case of the high-inclination $\left(\gtrsim 60^{\circ}\right)$ low-mass X-ray binaries (LMXBs), periodic decreases in the X-ray flux (X-ray dips) extending over $10 \%-30 \%$ of their orbital phase have been observed (e.g., White et al. 1995). In these cases, it is generally accepted that the X-ray variations are due to absorption in the "bulge" at the edge of the accretion disk, where the accretion stream from the Roche-lobe filling companion star impacts the accretion disk (White \& Holt 1982; Bisikalo et al. 2005). Such variations have been predominantly observed in the X-ray 
light curves of high-inclination neutron star LMXBs, viz., XB 1916-053 (e.g., Boirin et al. 2004), XB 1254-690 (e.g., Smale et al. 2002), and EXO 0748-676 (Church et al. 1998).

On the other hand, sharp drops in the X-ray flux lasting less than $1 \%$ of the orbital phase have been observed in a small sample of accreting black hole binaries. These include the LMXB GRO J1655-40 (Kuulkers et al. 1998, 2000) and the high-mass X-ray binary (HMXB) Cygnus X-1 (Balucińska-Church et al. 2001; Feng \& Cui 2002). In the case of GRO J1655-40, the sharp dips last a very small fraction of the orbital period (a few minutes compared to its orbital period of 2.62 days) and are confined between the orbital phases of 0.7 and 0.9 (phase 0 corresponds to the superior conjunction of the X-ray source, i.e., when the companion star is in front of the black hole with respect to our line of sight). The short duration of these dips suggests that the absorbing medium is likely filamentary in nature. Based on three-dimensional numerical simulations of the accretion stream impacting the disk in compact binaries (Armitage \& Livio 1998), Kuulkers et al. (2000) suggested that the likely scenario operating in GRO J1655-40 is that the accretion stream from the Roche-lobe overflow of the stellar companion splashes onto the disk rim, creating a local distribution of material above and below the plane of the disk. Also, the numerical work by Armitage \& Livio (1998) predicts that the sharp dips should occur preferentially around the orbital phase of 0.8 (the stream-disk impact site), similar to those observed in GRO J1655-40 (Kuulkers et al. 2000). This suggested that the absorber in the case of GRO J1655-40 is a local distribution (between orbital phases of 0.7-0.9) of clumps above and below the accretion disk and is then somewhat different compared to the dipping in neutron star LMXBs.

The HMXB Cygnus X-1 exhibits two types of "sharp" X-ray dips (type A and type B as classified by Feng \& Cui 2002). The type-A dips are energy-dependent, i.e., accompanied by an increase in the hardness ratio while the type-B dips are energy independent, i.e., no evidence for an increase in the hardness ratio. While the type-A dips are preferentially distributed roughly about the superior conjunction of the X-ray source, type-B dips occur randomly over the binary orbit. The type-A dips are attributed to being produced due to absorption by density enhancements (clumps or "blobs," in the vernacular) in an inhomogeneous wind from the companion star. On the other hand, type-B dips are suggested to be caused by partial covering of an extended X-ray source by an opaque screen (Feng \& Cui 2002). Numerical simulations of the accretion flow of wind-fed systems including HMXBs have shown that the tidal force from the compact object can distort the companion star and give rise to a focused wind in the direction of the compact source (Blondin et al. 1991). This tidal wind then develops into a Roche-lobe overflow as the surface of the stellar companion reaches its equipotential surface. The Coriolis force deflects the accretion stream such that it does not directly impact the compact companion but goes around it (see Figure 7 of Blondin et al. 1991). The density in these tidal streams can be as high as 20-30 times the ambient density and therefore, in principle, can serve as an opaque absorber of the X-rays (see also Balucińska-Church et al. 2001). Furthermore, these simulations predict that systems with dominant tidal streams should show evidence for pronounced dipping around the orbital phase of $\sim 0.6$. A study of the distribution of the sharp X-ray dips from Cygnus X-1 as a function of orbital phase has shown that there are indeed two peaks: the primary peak corresponds to phase 0 , i.e., superior conjunction of the X-ray source, and the secondary peak to a phase of 0.6 (Balucińska-Church et al. 2001).

In summary, two types (based on the duration of the individual dips) of periodic/quasi-periodic X-ray modulations have been observed in accreting X-ray binaries: (1) broad dips lasting $10 \%-30 \%$ of the orbital period have been seen in a sample of neutron star LMXBs and (2) sharp dips lasting less than $1 \%$ of the orbital period have been observed from the black hole LMXB and HMXB, GRO J1655-40 and Cygnus X-1, respectively. It is important to note that the location of the absorber is different in the neutron star LMXBs (bulge at the edge of the accretion disk) compared with GRO J1655-40 (clumps above the accretion disk) and Cygnus X-1 (accretion stream).

Here we report on a study of the X-ray monitoring data from NGC 5408 X-1 obtained with the Swift X-Ray Telescope (XRT). In particular, we present evidence for sharp dips in the $\mathrm{X}$-ray light curve and interpret the observed variability in the context of binary motion. The paper is arranged as follows. In Section 2 we discuss the details of the Swift/XRT data used for this work. In Section 3 we study the long-term $(\sim$ a few hundred days) timing behavior of the source. In particular, we report the detection of the quasi-periodic, sharp X-ray dips along with the smooth, quasi-sinusoidal X-ray modulation. Both modulations are consistent with the dipping phenomena seen in accreting galactic X-ray binaries. However, to avoid confusion and to be able to clearly distinguish between the two kinds of modulations, we will refer to the sharp X-ray dips as simply dips or sharp dips while we refer to the smooth component as the smooth quasisinusoidal X-ray modulation. In Section 4 we present spectral evidence for a change in the physical properties of the system. We also study the spectral differences between the sharp dips and the other portions of the data. We discuss the implications of our results on the orbital motion of the X-ray source in Section 5. We summarize our work in Section 6.

\section{SWIFT/XRT OBSERVATIONS}

The XRT onboard Swift began monitoring the ULX NGC 5408 X-1 in 2008 April as part of an approved Cycle 4 program (PI: Strohmayer). We include in our analysis all the observations obtained since the beginning of that program through 2011 August 30. The observing cadence has varied over this time span of $\approx 3.4 \mathrm{yr}$ (1240 days), but on average the source was observed, when viewable, for a few ks once every four days. Gaps in the coverage due to Swift observing constraints occurred from 2008 September 26-2008 December 16 (81 days), 2009 September 25-2009 December 15 (81 days), 2010 September 26-2009 December 20 (85 days), and 2011 March 27-2011 May 1 (35 days). This provided a total of 305 pointed observations with a cumulative exposure of $\approx 500 \mathrm{ks}$ distributed over a temporal baseline of $\approx 1240$ days.

All the observations were carried out in the photon counting data mode. We began our analysis with the level-1 raw XRT event files (data as stored in the Swift archive). Each of the event files was reduced with the standard xrtpipeline data reduction tool. One crucial consideration during the reduction process was to mitigate the impact of bad pixels/columns on the XRT CCD. When a source is positioned on such pixels, it can lead to an incorrect measurement of the flux. Furthermore, the bad pixels can result in an erroneous estimate of the response (effective area) of the instrument. The University of Leicester's XRT data analysis Web site (http://www.swift.ac.uk/analysis/xrt/exposuremaps.php) provides a detailed discussion of this problem. The solution is to 
All Photons in the energy range of $0.3-8.0 \mathrm{keV}$

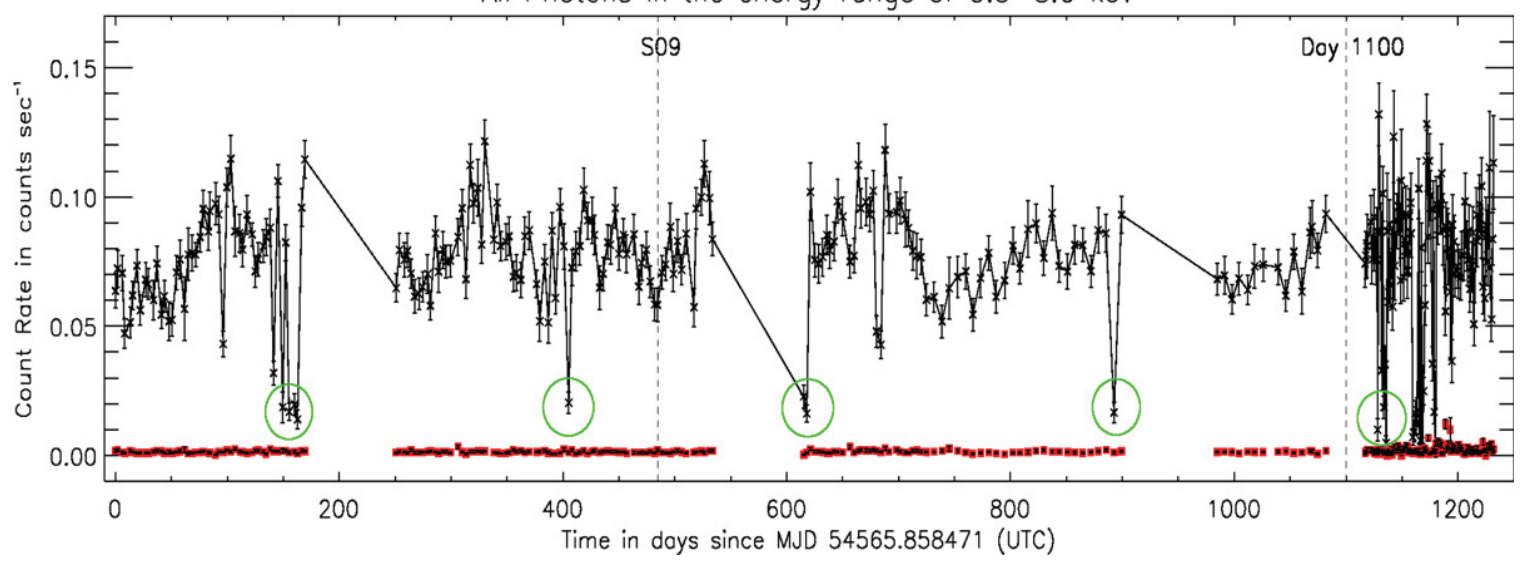

Figure 1. Swift/XRT X-ray light curve of the ULX NGC 5408 X-1 in the $0.3-8.0 \mathrm{keV}$ energy band. The background count rates with their respective error bars are also shown (red points and black error bars within). The most prominent feature of the light curve is the quasi-periodic recurrence of the dips. These are highlighted with green circles. The vertical line labeled as S09 marks the last observation used by Strohmayer (2009). The vertical line labeled as "day 1100" corresponds to the approximate epoch at which the physical properties of the system may have changed (see Section 4.1). Time zero corresponds to MJD 54565.85847 (UTC).

create exposure maps that account for the presence of bad pixels. We used xrtexpomap (xrtpipeline with the qualifier xrtexpomap = yes) to create exposure maps for each of the individual observations. These exposure maps were then used to correct the light curves (using xrtlccorr) and the ancillary response files (effective area; using xrtmkarf) of each of the observations.

As recommended in the XRT's user guide, we only used events with grades $0-12$ for further processing. We then used $X S E L E C T$ to extract light curves and spectra from the individual observations. We extracted source light curves and spectra from a circular region of radius $47^{\prime \prime} .1$ centered around the source. This particular value was chosen to include roughly $90 \%$ (at $1.5 \mathrm{keV}$ ) of the light from the source (estimated from the fractional encircled energy of the XRT). A background region, free of other sources, was extracted in a nearby region. Given the low individual exposure times, to better estimate the background, we chose a circular region of twice the radius of the source, i.e., four times the source area. The same source and background region was used for all the 305 observations. We present results from detailed timing and spectral analysis in the following sections.

\section{RESULTS: TIMING ANALYSIS}

This section is divided into four parts: (1) we show the complete $(\approx 1240$ day temporal baseline) Swift/XRT X-ray (0.3-8.0 keV) light curve of NGC 5408 X-1, highlighting the most prominent features, (2) we compute periodograms (Lomb-Scargle) of two different portions of this complete light curve (one with strong quasi-sinusoidal X-ray modulation and the other with apparently weaker modulation), (3) we estimate the distribution of dips with orbital phase and also construct an epoch folded light curve, and finally (4) we study the energy dependence of the quasi-sinusoidal modulation and the dips.

\subsection{X-Ray Light Curve}

For each observation, we combined all the data to obtain an average source and background count rate. The light curve of NGC $5408 \mathrm{X}-1$ in the $0.3-8.0 \mathrm{keV}$ energy range is shown in Figure 1. The background count rate in the same energy range is also shown (red data points with black error bars). The background is almost always negligible compared to the source count rate. Roughly $40 \%$ of these observations, i.e., the first 113 observations of the present data set, were analyzed by S09. The vertical line marked as S09 indicates the end of the observations used by S09 (see Figure 1). S09 reported a 115.5-day period in this data and concluded that it likely represents the orbital period of the binary. The additional data provide new insights into the system. A prominent feature of the full light curve is the quasi-periodic recurrence of deep, sharp X-ray dips (though with non-zero X-ray intensity during the dip minima). These dips are highlighted with green circles in Figure 1. With the available temporal baseline of $\approx 1240$ days, we detected five epochs of dips that recur roughly every 243 days. The approximate time intervals between the dips are 251 days, 211 days, 276 days, and 235 days.

\subsection{Timing Evidence for Weakening of the 115-Day Modulation}

In this section, we present evidence for weakening of the 115.5-day quasi-sinusoidal X-ray modulation during the second half ( $\approx$ last 600 days) of the light curve shown in Figure 1 . We started our analysis by dividing the light curve into roughly two equal segments. We then directly compared the Lomb-Scargle periodograms (Scargle 1982; Horne \& Baliunas 1986) of the two segments. We used all the photons in the $0.3-8.0 \mathrm{keV}$ energy range for this analysis. The two periodograms with their respective light curves are shown in Figure 2. The top left panel of the plot shows the light curve of the first half of the complete light curve (first half of Figure 1) and the top right panel shows the Lomb-Scargle periodogram of this segment with confidence limits overlaid. The bottom left panel shows the light curve of the second half of Figure 1 (note the $x$-axis) and the bottom right panel shows the periodogram of the second half of the complete light curve. The highest peak in the periodogram of the first half of the light curve (top right panel of Figure 2) corresponds to a period of $112.6 \pm 4$ days. This is consistent with the value reported by S09 (also see Han et al. 2012). Furthermore, such a peak is not evident in the periodogram of the second half of the data. We note that the reason for this difference is unlikely to be purely statistical as the two portions of the light curve have comparable signal-to-noise ratios, temporal baseline ( $\approx 600$ days each), and sampling rate (with the exception of the last continuous segment of the light curve which has a higher sampling rate of $\approx$ once per day). It is very likely that the drop in 

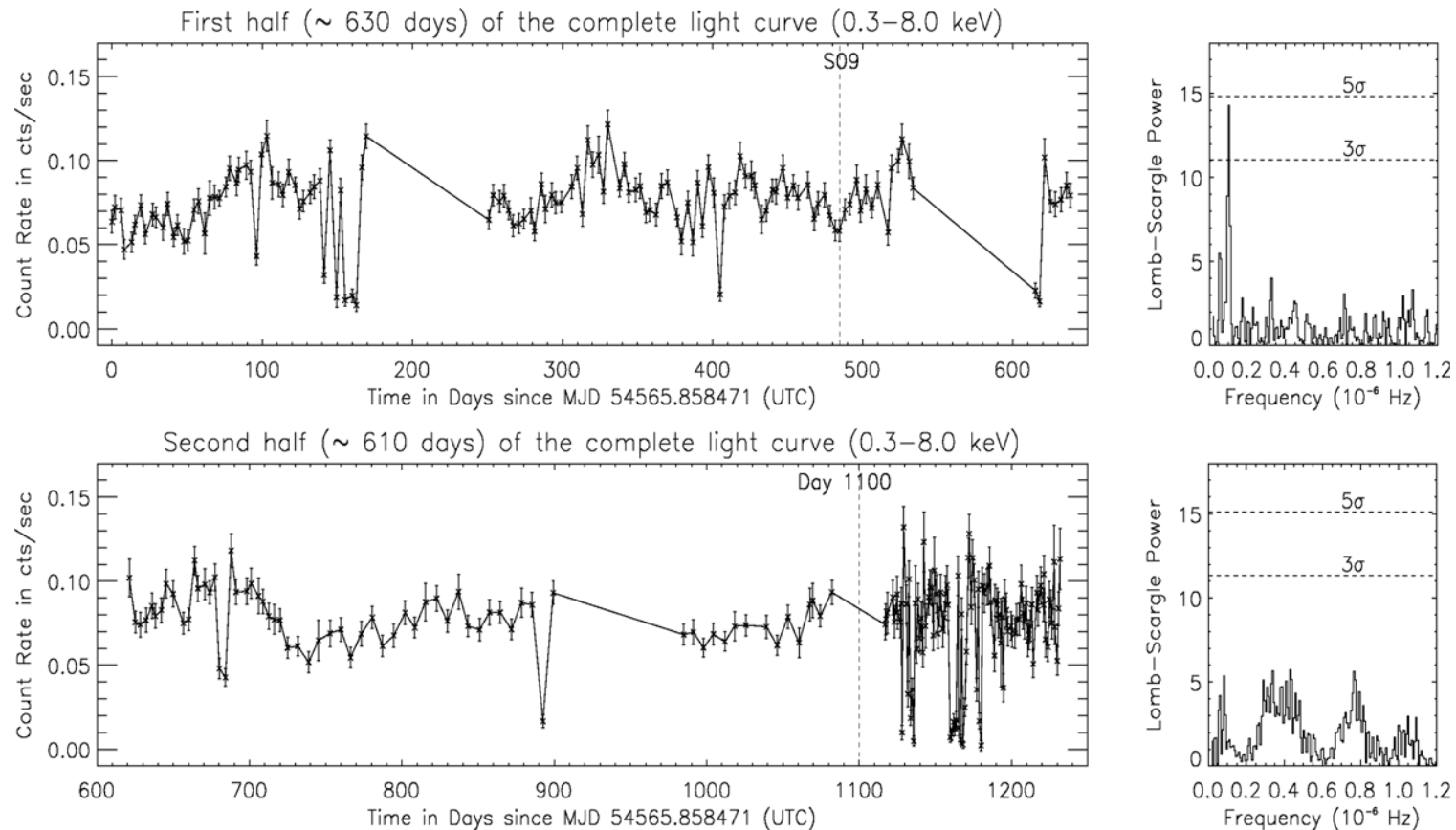

Figure 2. Top left panel: first half ( $\approx 630$ days) of the complete Swift/XRT X-ray light curve of the ULX NGC 5408 X-1 in the energy range $0.3-8.0$ keV. Top right panel: the Lomb-Scargle periodogram of the first half of the Swift/XRT X-ray light curve of NGC 5408 X-1 (top left panel). The highest peak corresponds to $112.6 \pm$ 4 days. Bottom left panel: second half $(\approx 610$ days) of the Swift/XRT X-ray light curve of the ULX NGC 5408 X-1 in the energy range $0.3-8.0 \mathrm{keV}$. Note the erratic dipping in the last continuous segment of the data. Bottom right panel: the Lomb-Scargle periodogram of the second half of the complete Swift/XRT X-ray light curve of NGC 5408 X-1 (bottom left panel). Note the difference between the two periodograms: there is no statistically significant period detected during the second half of the light curve.

the amplitude of the modulation in the second half of the light curve is physical.

\subsection{Dip Distribution and the Folded Light Curve}

We have detected a total of 27 dips (see Figure 1). Each of these dips was assigned a phase assuming a period of 243 days, the average time interval between successive epochs of dipping. A normalized distribution of the phases of these dips is shown in Figure 3. A normalized distribution was obtained by dividing each value by the number of times each phase bin was observed. This was done to eliminate the bias of preferentially sampling certain phase bins. Since the absolute orbital phase of the system is not known, we arbitrarily assign the zero phase to correspond to the start of the monitoring observations. One can see that the dip phase distribution is roughly Gaussian with a width (FWHM) of $\approx 0.24$.

Another analysis tool to understand the nature of the modulations is epoch folding of the light curve. In the case of NGC 5408 $\mathrm{X}-1$, we have detected five epochs of sharp dips. If these are associated with the orbital period of the binary then the implied period is likely in the range $243 \pm 23$ days. The variance (23 days) on this value is rather large, thus, the precise value of the orbital period to use for folding the data is not known. Therefore, we used values between 220 and 270 days and then chose the value that resulted in the highest modulation amplitude. This value is 230 days. Furthermore, it was noted previously that the smooth, quasi-sinusoidal modulation disappears/weakens in the latter half of the data. Therefore, we used only the portion of the complete light curve that shows strong evidence for both components, i.e., the first $\approx 700$ days of the data. The epoch folded light using all the photons in the $0.3-8.0 \mathrm{keV}$ X-ray band is shown in Figure 4, and one can clearly see both modulations.

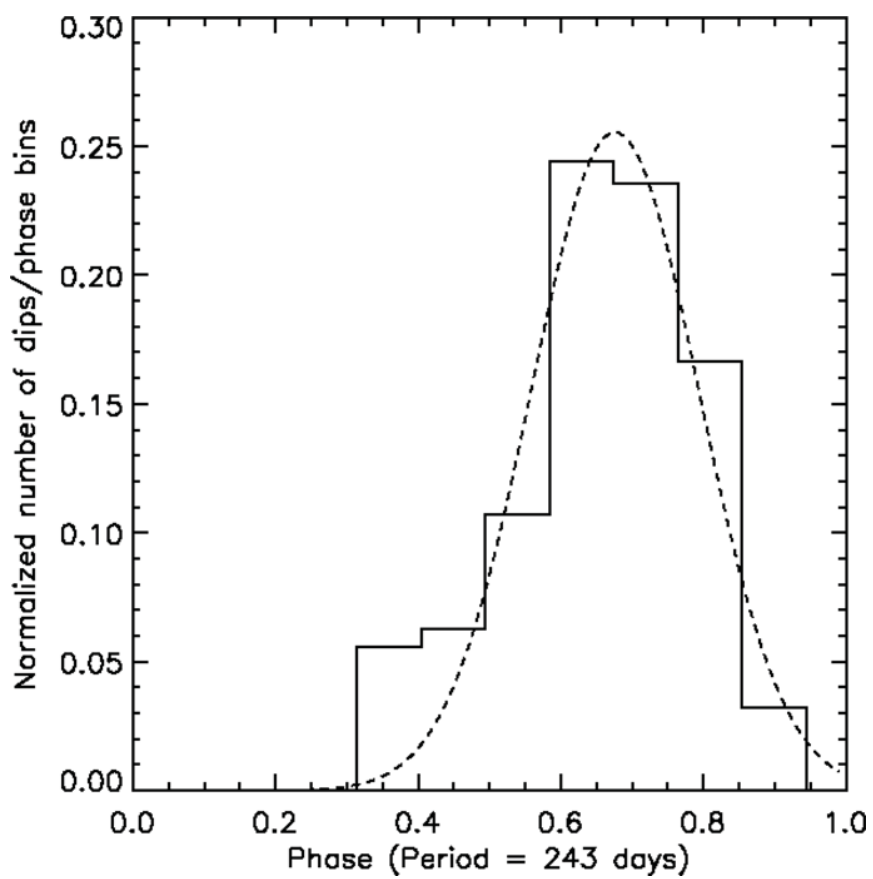

Figure 3. Frequency of the sharp dips as a function of the orbital period (243 days). It is normalized by dividing each value by the number of times each phase bin was observed. A total of 27 dips were detected. Eleven phase bins were used for the above distribution. The best-fit Gaussian is also shown with a dashed line.

\subsection{Energy Dependence of the X-Ray Modulations \\ 3.4.1. Energy-dependent Quasi-sinusoidal Modulation}

Using only part of the present data set, S09 reported that the amplitude of the quasi-sinusoidal X-ray modulation was energy 


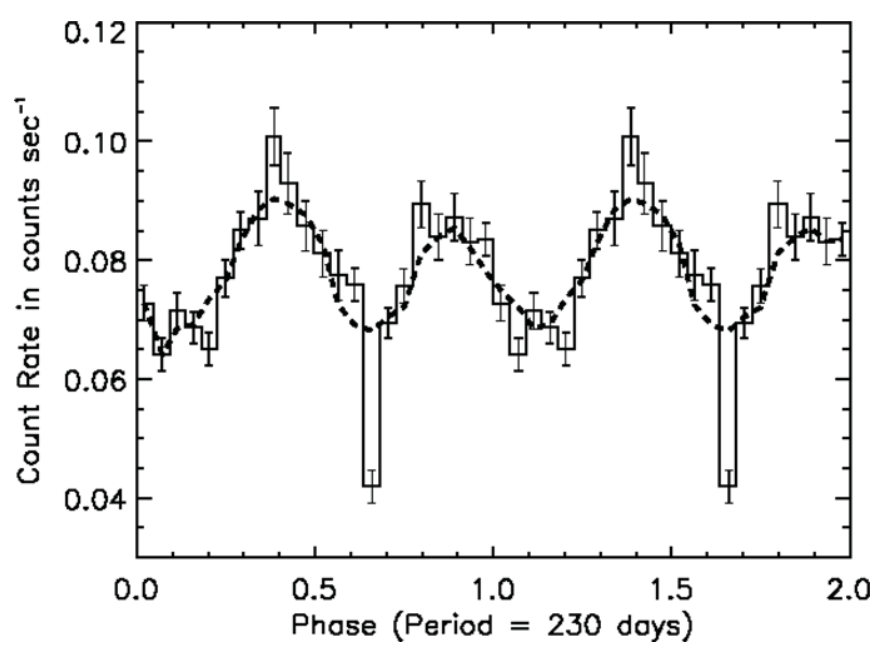

Figure 4. Epoch folded (period $=230$ days) X-ray $(0.3-8.0 \mathrm{keV})$ light curve of NGC 5408 X-1. A total of 22 phase bins per cycle were used and two cycles are shown for clarity. Also overplotted is a running average curve to guide the eye. Each point is an average of five neighboring bins. Both the smooth components (period of 115.5 days) and the sharp dips (quasi-period of $243 \pm 23$ days) can be clearly seen.

dependent, with the modulation amplitude decreasing with increasing energy. Here we attempt to systematically quantify the variability as a function of energy. The procedure we carry out is as follows. We obtain folded light curves in different energy bands. More specifically, keeping the upper limit of the bandpass constant at $8.0 \mathrm{keV}$, we vary the lower limit of the bandpass from 0.3 to $2.0 \mathrm{keV}$. Again, since the 115.5-day modulation weakens during the latter half of the monitoring we used only the first $\approx 700$ days of data. A few folded profiles along with the best-fitting model (solid line) are shown in Figure 5. Two cycles are shown for clarity. Here we used only 13 phase bins per cycle as our goal is to model only the overall quasisinusoidal modulation and not to study the subtle features within the modulation profiles.

To each of these profiles we fit a model that includes two Fourier components (the fundamental and the first harmonic), i.e., $I=A+B \sin 2 \pi\left(\phi-\phi_{0}\right)+C \sin 4 \pi\left(\phi-\phi_{1}\right)$. All the fits give acceptable values of reduced $\chi^{2}(\approx 1$ with 8 degrees of freedom (dof)) (Table 1). The fractional amplitude for such a model is defined as $f_{\text {amplitude }}=(\max (I)-\min (I)) /(\max (I)+\min (I))$, and is an indicator of the amount of variability in the source in the given energy range. Figure 6 shows the variation of the fractional amplitude (y-axis) as a function of the lower limit of the bandpass considered ( $x$-axis). Clearly, the fractional amplitude of the X-ray modulation is dependent on the energy range under consideration. The amplitude decreases with increasing energy. These results are consistent with those reported by S09.

\subsubsection{Energy Independent or Dependent Sharp Dips?}

To study the energy dependence of the dips, we first extracted the hardness ratio during these dips and elsewhere. We define this as the ratio of the count rate in the hard band $(1.0-8.0 \mathrm{keV})$ to the soft band $(0.3-1.0 \mathrm{keV})$. The top panel of Figure 7 shows the hardness ratio as a function of time (in days). The middle and bottom panels show the light curves in the soft $(0.3-1.0 \mathrm{keV})$ and the hard $(1.0-8.0 \mathrm{keV})$ band, respectively. Owing to the lower count rates during the dips, the error bars on the hardness ratio are large. It is not clear from these plots alone whether the dips from NGC 5408 X-1 are energydependent. To explore further we obtained an average hardness ratio of the dips before and after day 1100 . These values are $1.18 \pm 0.17$ and $0.95 \pm 0.19$, respectively. Within the error bars they are not only consistent with each other, but are also consistent with the average value of the non-dip observations. This suggests that the X-ray dips are largely energy independent.

\section{RESULTS: SPECTRAL ANALYSIS}

This section is divided into two parts: (1) we investigate the differences in the spectra derived from two different portions of the complete light curve, providing evidence for a change in the spectrum of the source and (2) we compare the average X-ray spectrum of the dips with the spectra derived from the rest of the observations.

\subsection{Spectral Evidence for a Change in the Absorption}

In this section we investigate the differences in the average spectra of the source before and after day 1100 . We chose this epoch because of the apparent increase in the frequency of dips compared to the earlier parts of the light curve. For this purpose we used all but the dip observations, i.e., the observations with count rate $(0.3-8.0 \mathrm{keV})$ lower than 0.045 counts $\mathrm{s}^{-1}$ were excluded. The number of counts in any individual observation is too low (a few $10 \mathrm{~s}$ of counts) to extract a meaningful spectrum. This necessitated combining the individual observations to obtain an average spectrum. We did this for all observations both preceding and following day 1100, resulting in two spectra for comparison. We used the FTOOL sumpha to combine the individual spectra. The two spectra in the X-ray energy range of 0.5-8.0 keV are shown in Figure 8. The average spectrum prior to day 1100 is shown in black while the data from after day 1100 is shown in red. Both these spectra were binned to ensure a minimum of 50 counts in each spectral bin.

To quantify the spectra we fit them with a model that is often used to describe the X-ray spectra of accreting black hole binaries: a multi-colored disk and a power law, all modified by photoelectric absorption. This same model has been used to fit the high-resolution $(\approx 100,000$ counts) XMM-Newton spectra of NGC 5408 X-1 (Strohmayer et al. 2007; Dheeraj \& Strohmayer 2012). We used the XSPEC (Arnaud 1996) spectral fitting package to fit all our spectra. In terms of XSPEC models, we used phabs*(diskpn + pow). The X-ray spectrum of the dips (green points in Figure 8) suffers from significant statistical uncertainty below $0.6 \mathrm{keV}$. Therefore, we used the energy range of $0.6-8.0 \mathrm{keV}$ for its spectral modeling. To be consistent across all the X-ray spectra (as we will be comparing them directly with each other) we chose the same energy range to model the two average, non-dip spectra. The results of the spectral fits are summarized in Table 2.

Even without detailed modeling it can be seen straightaway that there are significant differences between the two spectral below $1.5 \mathrm{keV}$ (black and red symbols in Figure 8). A closer look at the unfolded spectra reveals that the disk blackbody component dominates at energies below $2 \mathrm{keV}$ while the powerlaw component dictates the nature of the spectrum at higher energies; and the photoelectric absorption is stronger at lower energies. Therefore, the apparent differences between the two spectra can either be due to a change in the absorption column density (phabs) or to changes in the disk properties, viz., the disk temperature and/or the disk normalization. The quality of the data does not allow us to independently constrain each model's parameters. To break this degeneracy, either one of the diskpn model parameters (the disk temperature or the 

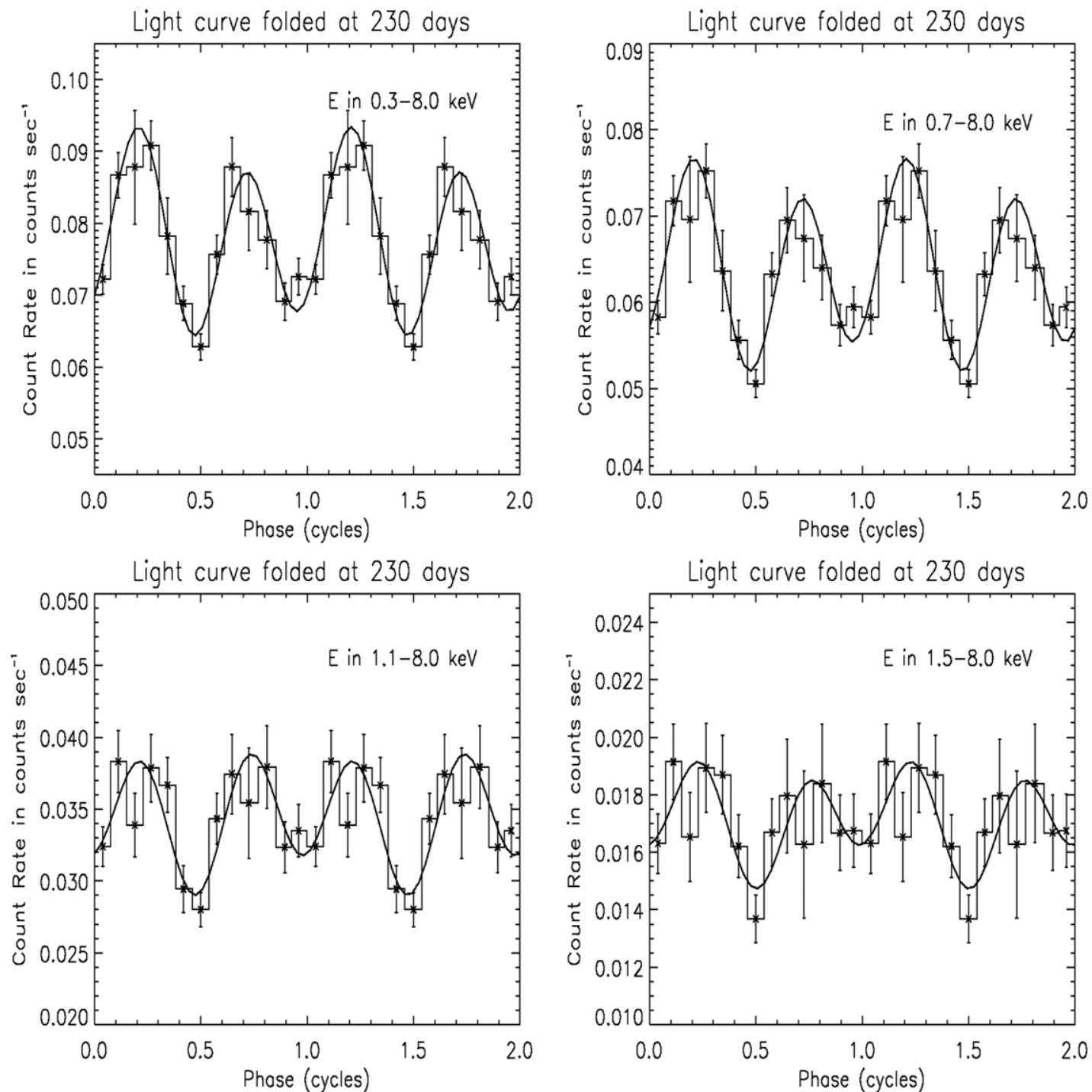

Figure 5. Epoch folded light curves of NGC $5408 \mathrm{X}-1$ in varying energy bands. The light curves in the energy ranges $0.3-8.0 \mathrm{keV}$, 0.7-8.0 keV, 1.1-8.0 keV, and $1.5-8.0 \mathrm{keV}$ are shown in the top left, top right, bottom left, and bottom right panels, respectively. In each case, we used 13 phase bins per cycle and two cycles are shown for clarity. To each of these profiles we fit a model that includes two Fourier components (the fundamental and the first harmonic), i.e., $I=A+B \sin 2 \pi\left(\phi-\phi_{0}\right)+C \sin 4 \pi\left(\phi-\phi_{1}\right)$. The best-fitting curve is overlaid (solid curve). Clearly, the modulation amplitude decreases with increasing energy.

Table 1

Summary of the Best-fitting Model Parameters of the X-ray Modulation Profiles of NGC 5408 X-1

\begin{tabular}{lcccrrr}
\hline \hline $\begin{array}{l}\text { Bandpass } \\
(\mathrm{keV})\end{array}$ & $A^{\mathrm{a}}\left(10^{-2}\right)$ & $B^{\mathrm{a}}\left(10^{-2}\right)$ & \multicolumn{1}{c}{$\phi_{0}^{1}$} & $C^{\mathrm{a}}\left(10^{-2}\right)$ & \multicolumn{1}{c}{$\phi_{1}^{1}$} & $f_{\text {amplitude }}^{\mathrm{b}}$ \\
\hline $0.3-8.0$ & $7.82 \pm 0.09$ & $3.57 \pm 0.13$ & $0.88 \pm 0.05$ & $-1.20 \pm 0.13$ & $1.34 \pm 0.01$ & $0.184 \pm 0.021$ \\
$0.5-8.0$ & $7.55 \pm 0.09$ & $3.76 \pm 0.14$ & $0.91 \pm 0.05$ & $-1.25 \pm 0.13$ & $0.84 \pm 0.01$ & $0.196 \pm 0.021$ \\
$0.7-8.0$ & $6.40 \pm 0.09$ & $2.87 \pm 0.11$ & $0.86 \pm 0.07$ & $-1.03 \pm 0.12$ & $0.84 \pm 0.01$ & $0.191 \pm 0.023$ \\
$0.9-8.0$ & $4.89 \pm 0.08$ & $1.71 \pm 0.08$ & $0.79 \pm 0.11$ & $0.68 \pm 0.11$ & $1.09 \pm 0.01$ & $0.163 \pm 0.025$ \\
$1.1-8.0$ & $3.45 \pm 0.06$ & $1.40 \pm 0.07$ & $0.70 \pm 0.11$ & $0.41 \pm 0.08$ & $0.60 \pm 0.01$ & $0.144 \pm 0.028$ \\
$1.3-8.0$ & $2.39 \pm 0.05$ & $1.21 \pm 0.05$ & $0.71 \pm 0.10$ & $0.28 \pm 0.07$ & $0.60 \pm 0.02$ & $0.147 \pm 0.032$ \\
$1.5-8.0$ & $1.71 \pm 0.04$ & $0.08 \pm 0.04$ & $0.81 \pm 0.12$ & $-0.16 \pm 0.06$ & $-0.13 \pm 0.03$ & $0.130 \pm 0.039$ \\
$1.7-8.0$ & $1.29 \pm 0.04$ & $0.08 \pm 0.04$ & $0.81 \pm 0.11$ & $-0.12 \pm 0.05$ & $-1.13 \pm 0.03$ & $0.139 \pm 0.045$ \\
$2.0-8.0$ & $0.90 \pm 0.03$ & $0.07 \pm 0.03$ & $0.78 \pm 0.10$ & $0.07 \pm 0.03$ & $0.61 \pm 0.04$ & $0.132 \pm 0.050$ \\
\hline
\end{tabular}

Notes.

${ }^{a}$ We fit the X-ray modulation profiles with a model consisting of two Fourier components. The mathematical form of the model is as follows:

$I=A+B \sin 2 \pi\left(\phi-\phi_{0}\right)+C \sin 4 \pi\left(\phi-\phi_{1}\right)$,

where $A$ is the mean count rate while $B$ and $C$ are the amplitudes of the fundamental and the first harmonics, respectively.

$\mathrm{b}$ This parameter is the fractional modulation amplitude and gives a quantitative measure of the amount of variation in the pulse profile. This is defined as follows:

$$
f_{\text {amplitude }}=\frac{I_{\max }-I_{\min }}{I_{\max }+I_{\min }} .
$$


Table 2

Summary of the Spectral Modeling of NGC 5408 X-1

\begin{tabular}{lccccccc}
\hline \hline \multicolumn{7}{c}{ phabs*(diskpn+pow) } \\
\hline Data Set & $n_{\mathrm{H}^{\mathrm{a}}}$ & $N_{\text {disk }^{\mathrm{b}}\left(\times 10^{-2}\right)}$ & $\Gamma^{\mathrm{c}}$ & $N_{\text {pow }}{ }^{\mathrm{d}}\left(\times 10^{-4}\right)$ & ${\text { Flux } 0.6-8.0 \mathrm{keV}^{\mathrm{e}}}^{\mathrm{e}}$ & Flux $_{\text {Disk }}{ }^{\mathrm{f}}$ & $\chi^{2} / \mathrm{dof}^{\mathrm{f}}$ \\
\hline $\begin{array}{c}\text { Average } \\
\text { spectrum } \\
\text { before day 1100 } \\
\text { Average } \\
\text { spectrum }\end{array}$ & $0.30_{-0.02}^{+0.02}$ & $1.57_{-0.27}^{+0.22}$ & $2.96_{-0.07}^{+0.07}$ & $7.69_{-0.48}^{+0.49}$ & $3.68_{-0.23}^{+0.21} \times 10^{-12}$ & $1.69_{-0.33}^{+0.29} \times 10^{-12}$ & $233 / 179$ \\
$\begin{array}{c}\text { after day 1100 } \\
\text { Average } \\
\text { dip } \\
\text { spectrum }\end{array}$ & $0.44_{-0.04}^{+0.04}$ & $4.27_{-0.86}^{+0.83}$ & $2.97_{-0.15}^{+0.17}$ & $8.64_{-1.19}^{+1.42}$ & $6.71_{-0.77}^{+0.70} \times 10^{-12}$ & $4.35_{-0.77}^{+0.89} \times 10^{-12}$ & $112 / 87$ \\
\hline
\end{tabular}

Notes. Best-fitting parameters for the phabs*(diskpn+pow) model are shown.

${ }^{a}$ Total column density of hydrogen along the line of sight including the Galactic extinction (in units of $10^{22} \mathrm{~cm}^{-2}$ ). We used the phabs model in XSPEC.

b The normalization of the disk component. We used the diskpn model in XSPEC. The inner radius of the disk was fixed at 6 GM $/ c^{2}$.

The disk temperature was fixed at $0.149 \mathrm{keV}$ to avoid parameter degeneracy (see the text).

c The photon index of the power law.

$\mathrm{d}$ The normalization of the power-law component. We used the pow model in XSPEC.

e The total unabsorbed X-ray flux (in units of erg $\mathrm{cm}^{-2} \mathrm{~s}^{-1}$ ) in the energy range of $0.6-8.0 \mathrm{keV}$.

${ }^{\mathrm{f}}$ The disk contribution to the total X-ray flux (in units of erg $\mathrm{cm}^{-2} \mathrm{~s}^{-1}$ ) in the energy range of $0.6-8.0 \mathrm{keV}$.

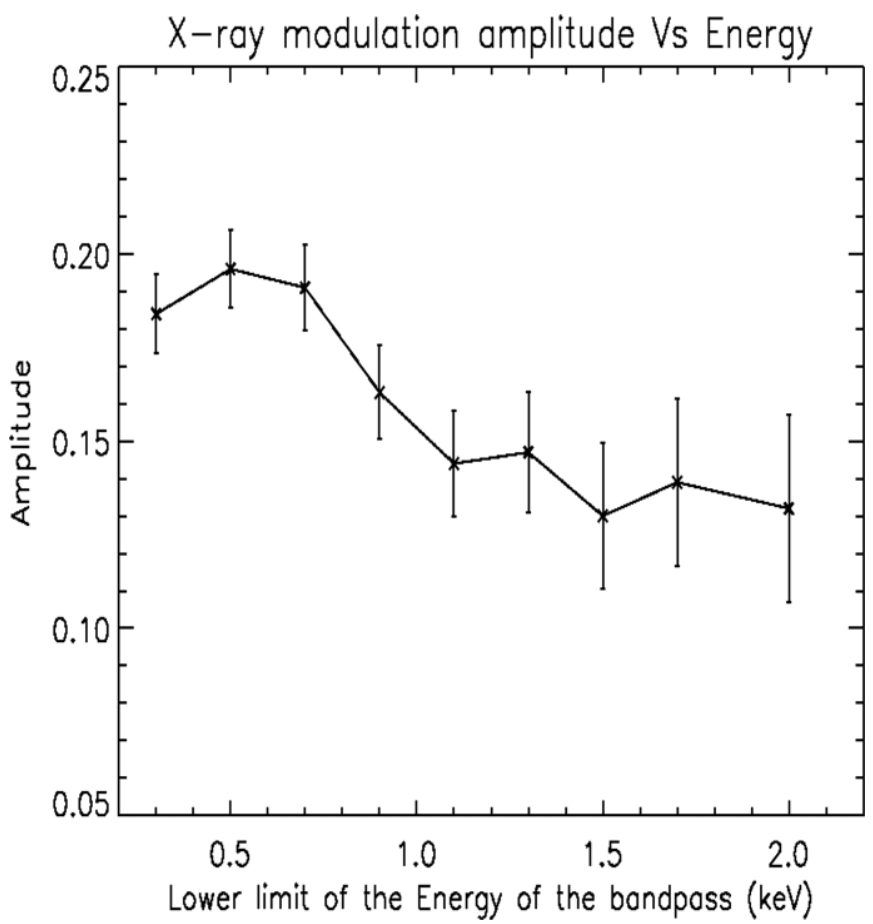

Figure 6. Fractional amplitude of the smooth component of the X-ray modulation as a function of the lower limit of the band pass is shown. The upper limit of the bandpass was fixed at $8 \mathrm{keV}$. The fractional amplitude of the mathematical form used to model the epoch folded light curves of NGC 5408 X-1 (Figure 4) is defined as $f_{\text {amplitude }}=(\max (I)-\min (I)) /(\max (I)+\min (I))$, where $I$ is the $\mathrm{X}$-ray count rate.

disk normalization) or the column density had to be frozen. A careful analysis of the spectra reveals stronger statistical evidence for a varying column density than varying disk properties.

To illustrate this we simultaneously, but independently, fit the model to both the spectra (without freezing any model parameters except for the inner radius of the accretion disk). We then obtain confidence contours $\left(\chi^{2}\right)$ between the column densities, the disk temperatures, and the disk normalizations of the spectra before and after day 1100. These confidence contours are shown in Figure 9. The top panel shows the confidence contours between the column density of hydrogen prior to day 1100 ( $x$-axis) and after day 1100 (y-axis). Similarly, the bottom left and the bottom right panel show the contours between the disk temperatures and the disk normalizations, respectively. In all the cases, the black, the red, and the green represent the $1 \sigma$, $2 \sigma$, and the $3 \sigma$ confidence contours, respectively. In each case, the diagonal line shows the locus of points where the value on the $X$-axis equals the value on the $Y$-axis.

The amount of deviation of the confidence contours from this line indicates the significance of the variation of a given parameter between the two epochs. In other words, the larger the deviation the stronger is the evidence for a change in the given parameter. Clearly, the statistical evidence for an increase in the column density after day 1100 is very strong (top panel: strong deviation from the straight line) compared to that for changes in the disk temperature (bottom left panel) and the disk normalization (bottom right panel). In addition, the source has been observed on multiple occasions with $X M M$ Newton over the past 10 years. The disk temperature on those occasions has remained roughly constant (Soria et al. 2004; Strohmayer \& Mushotzky 2009; Dheeraj \& Strohmayer 2012). Furthermore, in accreting galactic black hole binaries, changes in the accretion disk temperature are often accompanied by significant variations in the X-ray light curve (McClintock \& Remillard 2006). Clearly, this is not seen in the light curve of NGC $5408 \mathrm{X}-1$. These arguments suggest that the disk temperature has likely remained constant. Therefore, for subsequent analysis we assumed a constant disk temperature of $0.149 \mathrm{keV}$, the average value reported from the analysis of the high-quality XMM-Newton data (Dheeraj \& Strohmayer 2012). The model then gives a reasonable fit in both cases (before and after day 1100) with reduced $\chi^{2}$ of 1.3 (179 dof) and 1.3 ( 87 dof). The best-fitting model parameters for the two average spectra are shown in the first and the second row of Table 2, respectively.

The most prominent difference between the two spectra is the amount of photoelectric absorption. The total hydrogen column density before and after day 1100 was $0.30_{-0.02}^{+0.02} \times 10^{22} \mathrm{~cm}^{-2}$ 


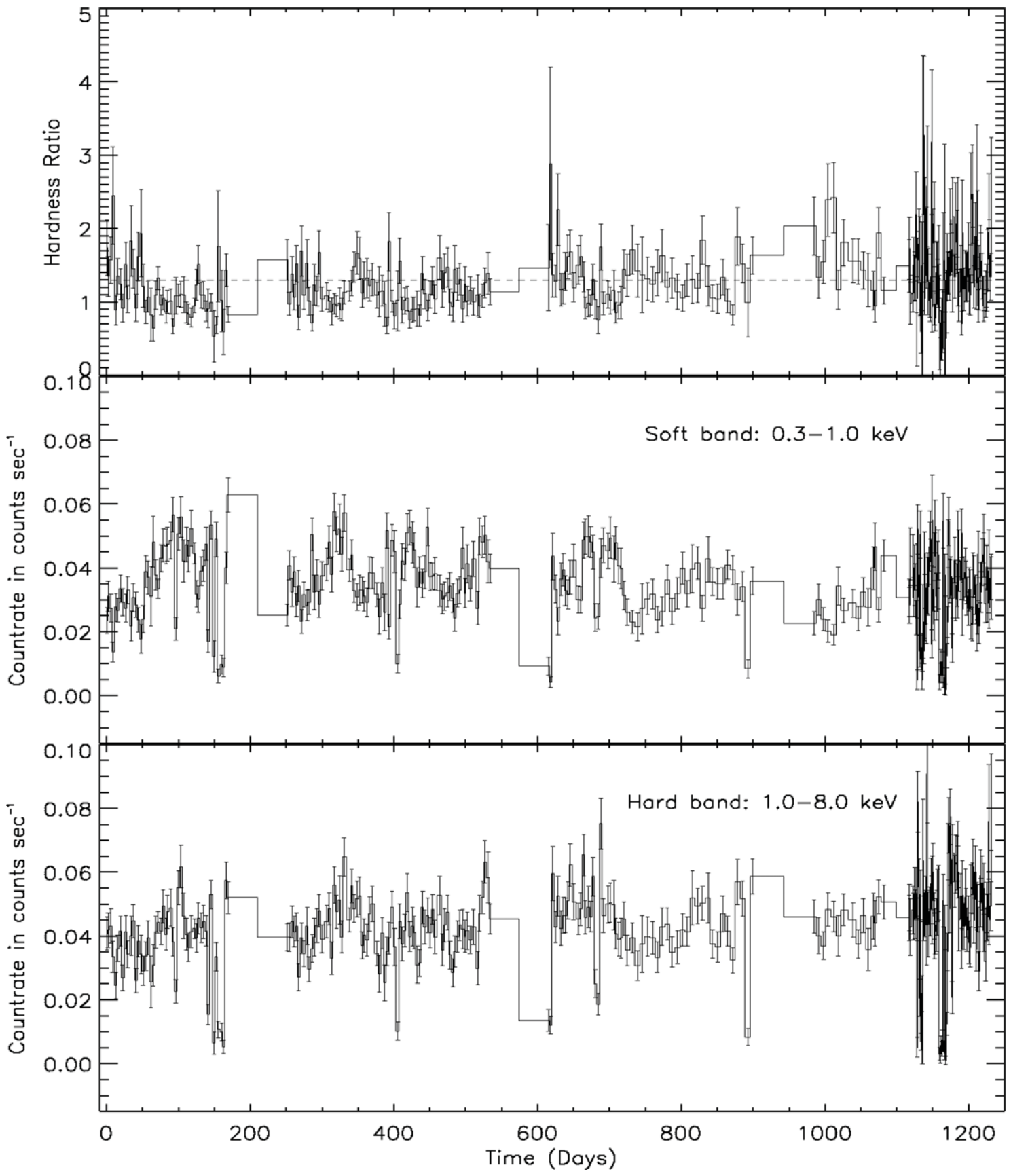

Figure 7. Top panel: time history of the hardness ratio (ratio of the count rate in the hard band $(1.0-8.0 \mathrm{keV})$ to the soft band $(0.3-1.0 \mathrm{keV})$ ). The mean value is indicated by the dashed line. Middle panel: the light curve of the source in the soft band, i.e., $0.3-1.0 \mathrm{keV}$. Bottom panel: the light curve of the source in the hard band, i.e., $1.0-8.0 \mathrm{keV}$.

and $0.44_{-0.04}^{+0.04} \times 10^{22} \mathrm{~cm}^{-2}$, respectively. That is, the latter $\mathrm{X}$-ray spectrum is more absorbed than the former. The estimated total unabsorbed flux in the $0.6-8.0 \mathrm{keV}$ range before and after day 1100 is $3.7_{-0.2}^{+0.2} \times 10^{-12} \mathrm{erg} \mathrm{cm}^{-2} \mathrm{~s}^{-1}$ and $6.7_{-0.8}^{+0.7} \times 10^{-12} \mathrm{erg}$ $\mathrm{cm}^{-2} \mathrm{~s}^{-1}$, respectively. This suggests a factor of two increase in the unabsorbed X-ray flux during the last continuous segment of the light curve. Another important thing to note is the fraction of the disk contribution to the total flux before and after day 1100. The values are $1.7_{-0.3}^{+0.3} \times 10^{-12} \mathrm{erg} \mathrm{cm}^{-2} \mathrm{~s}^{-1}$ and

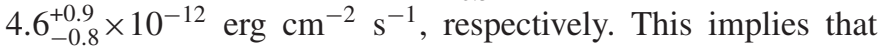
the disk fraction of the total X-ray flux $(0.6-8.0 \mathrm{keV})$ before and after day 1100 is $46 \% \pm 9 \%$ and $68 \% \pm 15 \%$, respectively. In other words, the latter spectrum appears to be more disk-dominated.

\subsection{Spectra During the Sharp Dips versus Elsewhere}

The dips detected here occur both prior to and after day 1100 (see Figure 1); and the individual dip observations have too few counts (a few $10 \mathrm{~s}$ ) to extract meaningful spectra. However, it was noted earlier that the average hardness ratios of both the dips before and after day 1100 are comparable, suggesting a plausible common physical origin. Therefore, we combined all the dip observations (both prior to and after day 1100) and obtained an average dip spectrum. This is shown in Figure 8 (green data points) along with the two other average spectra. The spectrum was binned to ensure a minimum of 25 counts in each spectral bin.

We began by fitting the dip spectrum with the same model $\left(\right.$ phabs* ${ }^{*}($ diskpn+pow $\left.)\right)$ used to fit the two spectra described in 


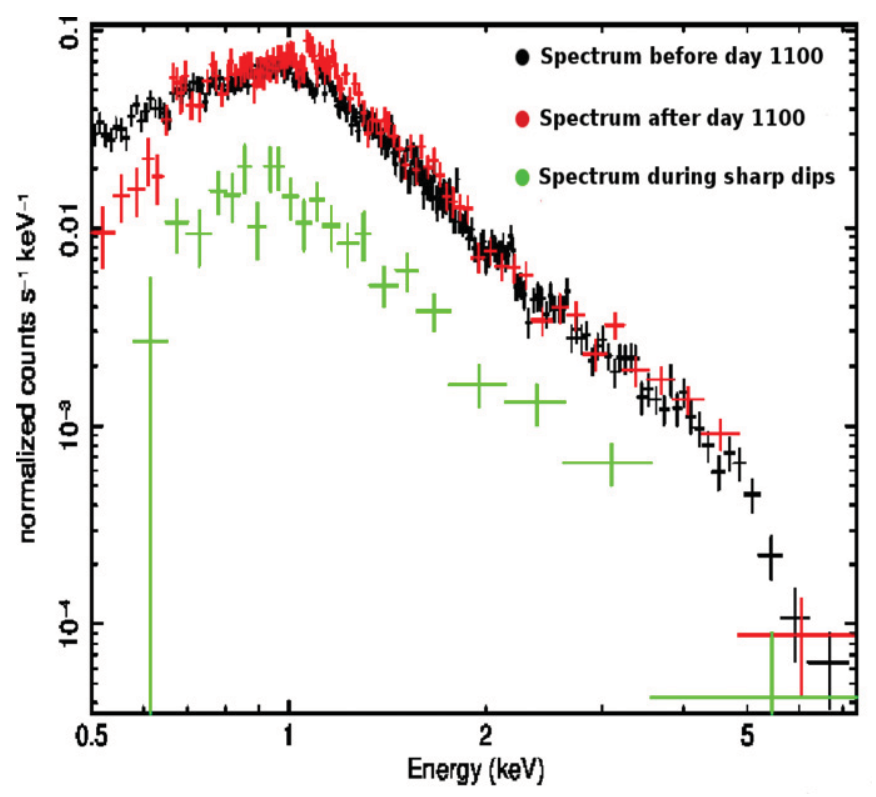

Figure 8. Swift/XRT spectra of NGC 5408 X-1 in the energy range $0.5-8.0 \mathrm{keV}$. The black points show the average spectrum prior to day 1100 (as marked by the vertical line in Figure 1). Shown in red is the average spectrum of the source after day 1100 epoch. There are clear differences between the two spectra, especially at lower energies (below $1.5 \mathrm{keV}$ ). The green data points denote the average spectrum during the sharp dips. The two spectra, i.e., the spectra before and after day 1100, are binned to ensure a minimum of 50 counts per spectral bin, while the dip spectrum is binned to ensure a minimum of 25 counts per spectral bin.

the previous section. This model gives a good fit with a reduced $\chi^{2}$ of 1.0 (17 dof). The best-fit parameters are shown in the last row of Table 2. We find that the best-fit parameters of the dip spectrum are consistent with the values for the two average non-dip spectra derived earlier. This is also consistent with the simpler hardness ratio analysis, i.e., the dips are consistent with being energy-independent.

The dip spectra of X-ray binaries are often characterized using more complex models involving either an absorbedplus-unabsorbed (hereafter, A+U) approach (e.g., Parmar et al. 1986; Courvoisier et al. 1986) or a progressive covering approach (e.g., Church et al. 1997, 1998; Church 2001). To explore the A+U approach we used the following model in XSPEC, phabs 1 *const $1 *($ diskpn+powerlaw $)+$ phabs $2 *$ const $2 *($ diskpn+powerlaw $)$, where the first and second terms represent the unabsorbed and absorbed components, respectively. In this case the only free parameters are the two normalization constants (const1 and const2) and the column density associated with phabs 2 . The parameters describing the shape of the spectrum are all fixed at the average best-fit values derived from the two non-dip spectra (first and second rows of Table 2). This model gives an acceptable fit with a reduced $\chi^{2}$ of 0.9 with 19 dof. The best-fit values of the normalization of the unabsorbed component, normalization of the absorbed component, and the column density of the absorbed component are 0.2 , $4.1 \times 10^{-4}$, and $2.7 \times 10^{22} \mathrm{~cm}^{-2}$, respectively. A simple interpretation in the context of this model is that during dips the direct $\mathrm{X}$-ray emission is completely obscured/absorbed, and what remains is largely scattered (in an energy-independent fashion) into our line of sight from more extended regions around the source.

Partial covering models have been quite successful in describing the spectral evolution with intensity during dipping (see, for example, Church et al. 1998). In this approach, the spectral evolution as dipping becomes stronger is accounted for by the progressive covering of, typically, one of the spectral components. The implication is that the progressively covered component is spatially extended compared to the other component. A perceived strength in favor of these models is that scattering-the details of which are not often specified in the $\mathrm{A}+\mathrm{U}$ approach-is not required. In the case of LMXB neutron star dippers it is the coronal (power-law) component that is progressively covered, whereas in some black hole systems successful modeling requires partial covering of the thermal (disk blackbody) component (Church 2001). We found that the dip spectrum of NGC 5408 X-1 could not be well described by progressive covering of either spectral component by itself, but that progressive covering of the sum of both components provided as good a fit as the $\mathrm{A}+\mathrm{U}$ modeling. The reason for this is two-fold: (1) the dip spectrum is consistent with being energy-independent, that is, it has the same shape as the non-dip spectra, and (2) both spectral components contribute a comparable amount to the total flux. Thus, it is very difficult to partially cover only one component and still maintain the same spectral shape. Partial covering of just the power-law component does a better job than covering of only the diskpn component, but in each case the fits are not as good as the $\mathrm{A}+\mathrm{U}$ case. Covering of the sum of the components provides a good fit. The implication in this case is that the emission components are extended but co-spatial. The dipping then corresponds to complete obscuration of about four-fifths of the extended source (in an average sense over all dips).

The conclusion from either modeling framework is that an absorbed component is not seen directly, only an unabsorbed component. Finally, we note that due to the limited statistical quality of the dip spectrum and the fact that only a single dip spectrum could be meaningfully extracted, it is difficult to be more precise about the exact physical nature of the dipping.

\section{DISCUSSION}

In this article, we have studied the long-term X-ray variability of the ULX NGC 5408 X-1 using 1240 days of Swift/XRT monitoring data. In addition to the periodic (115.5 days), smooth modulation reported by S09, the source also exhibits quasiperiodic sharp dips that occur on average every $243 \pm 23$ days, roughly twice the 115.5-day period. As discussed in Section 1, such orbit-phase-dependent X-ray dipping has been well studied in the case of accreting X-ray binaries and is usually ascribed to periodic obscuration of the X-ray source by an intervening medium, viz., a bulge at the edge of the accretion disk, the wind from the companion star, or the accretion stream. In those cases, dipping has been shown to be an excellent tracer of the orbital motion of the binary. Given that NGC 5408 X-1 is very likely an accreting black hole system, it is conceivable that a similar phenomenon causes the observed dipping. If that were indeed the case, then the detected period of $243 \pm 23$ days likely represents the orbital motion of the X-ray binary.

NGC 5408 X-1 is the only known ULX system that exhibits two types of dipping behavior, smooth and sharp, simultaneously. The basic properties of the smooth component are: (1) it occupies a significant portion $(\approx 50 \%)$ of the putative orbital cycle of $243 \pm 23$ days (see Figure 4), (2) it is energydependent with the modulation amplitude decreasing with increasing energy (see Figures 5 and 6), and (3) the modulation amplitude is variable, i.e., the smooth component modulation is weaker during the second half of the Swift monitoring data (see 

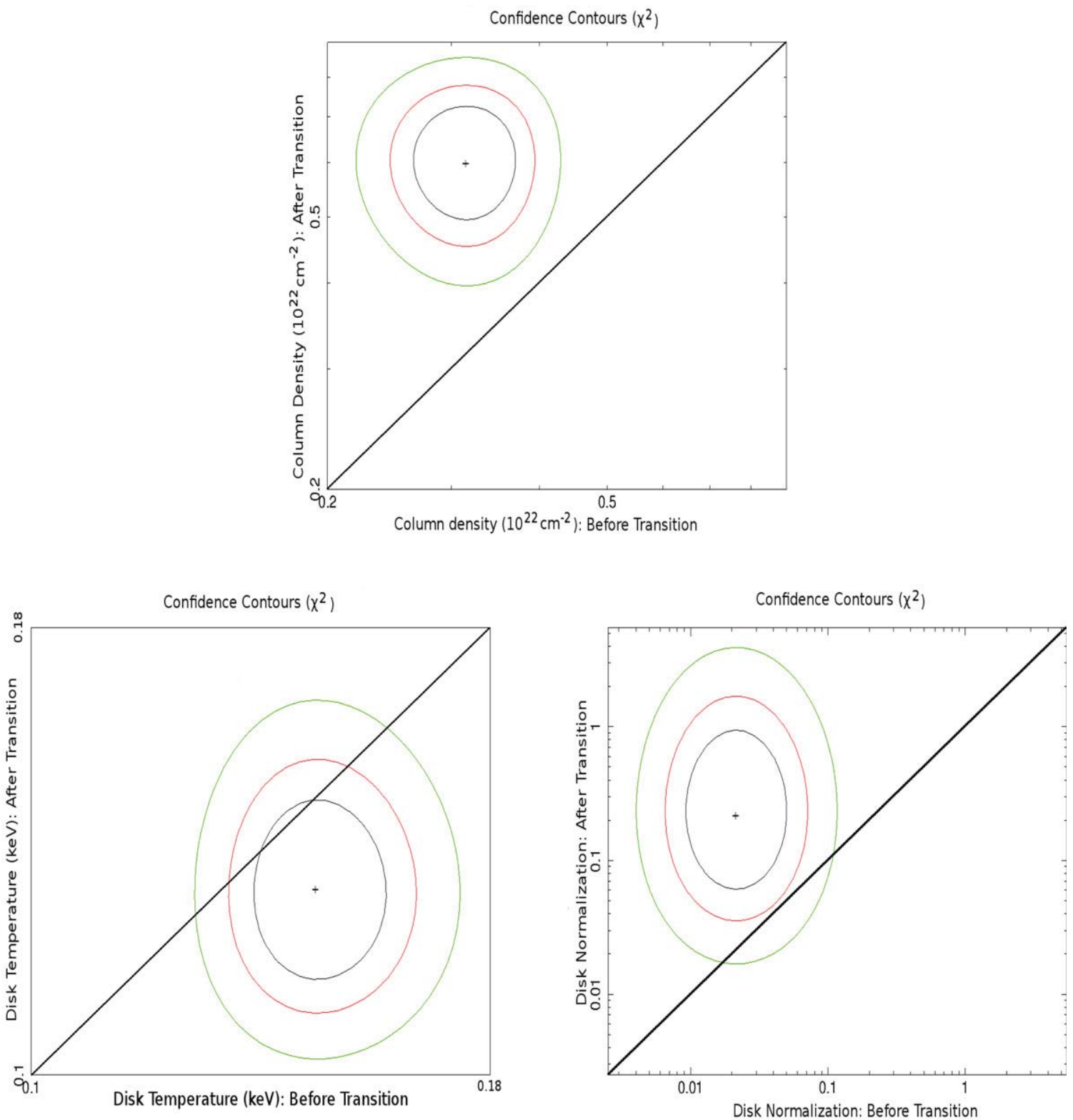

Figure 9. Top panel: the confidence contours of the total hydrogen column densities along the line of sight $\left(n_{\mathrm{H}}\right)$ before $(X$-axis) and after $(Y$-axis) day 1100 . Bottom left panel: the confidence contours of the disk temperatures before ( $X$-axis) and after ( $Y$-axis) day 1100 . Bottom right panel: the confidence contours of the disk normalizations before ( $X$-axis) and after ( $Y$-axis) day 1100. The black, red, and green curves in each panel represent the $1 \sigma, 2 \sigma$, and $3 \sigma$ confidence contours. Contours were obtained from fits to the non-dip spectra in both cases. The straight line in each case represents the locus of all points with $X=Y$. For a given parameter, if the contours do not overlap with this line then that is a strong indication for a change in the parameter value.

Figure 2). On the other hand, the sharp X-ray dips detected from NGC 5408 X-1 are not strictly periodic and are distributed over an orbital phase of $\approx 0.24$ (see Figure 3 ). In addition, they are consistent with being energy-independent (see Figure 8 and Table 2). We now discuss these observed properties within the framework of an accreting X-ray binary scenario.

\subsection{Roche-lobe Accretion: Eccentric Accretion Disk and Stream-Disk Interaction}

Assuming that the recurrence period of the X-ray dips is close to the orbital period of a Roche-lobe filling binary, the density of the companion star in NGC $5408 \mathrm{X}-1$ can be constrained using the formula, $\rho \simeq 0.2\left(P_{\text {days }}\right)^{-2} \mathrm{~g} \mathrm{~cm}^{-3}$ (Frank et al. 2002). For a period $\left(P_{\text {days }}\right)$ of 243 days, this would imply that the companion star has a mean density of $3.4 \times 10^{-6} \mathrm{~g} \mathrm{~cm}^{-3}$. This value is consistent with a recent study by Grisé et al. (2012), who extracted the UV/optical/NIR spectral energy distribution (SED) of the optical counterpart of NGC 5408 X-1. They find that the SED is consistent with a massive B0I super-giant star. Numerical hydrodynamical simulations of stream-fed accretion in low-mass ratio binaries show that the accretion disk can be tidally distorted (Armitage \& Livio 1996). This leads to the formation of a secondary bulge in the disk in addition to the primary bulge at the stream-disk impact site. In other words, tidal effects can lead to the formation of two broad bulges above the disk mid-plane along the outer rim of the accretion disk, separated by an orbital phase of $\approx 0.5$ (see Figure 5 of Armitage \& Livio 1996). This process may explain the observed modulations in NGC $5408 \mathrm{X}-1$. The modulation profile of the smooth component, including the phase separation between the two peaks, is in accord with the predicted X-ray variations from 


\section{Top View}
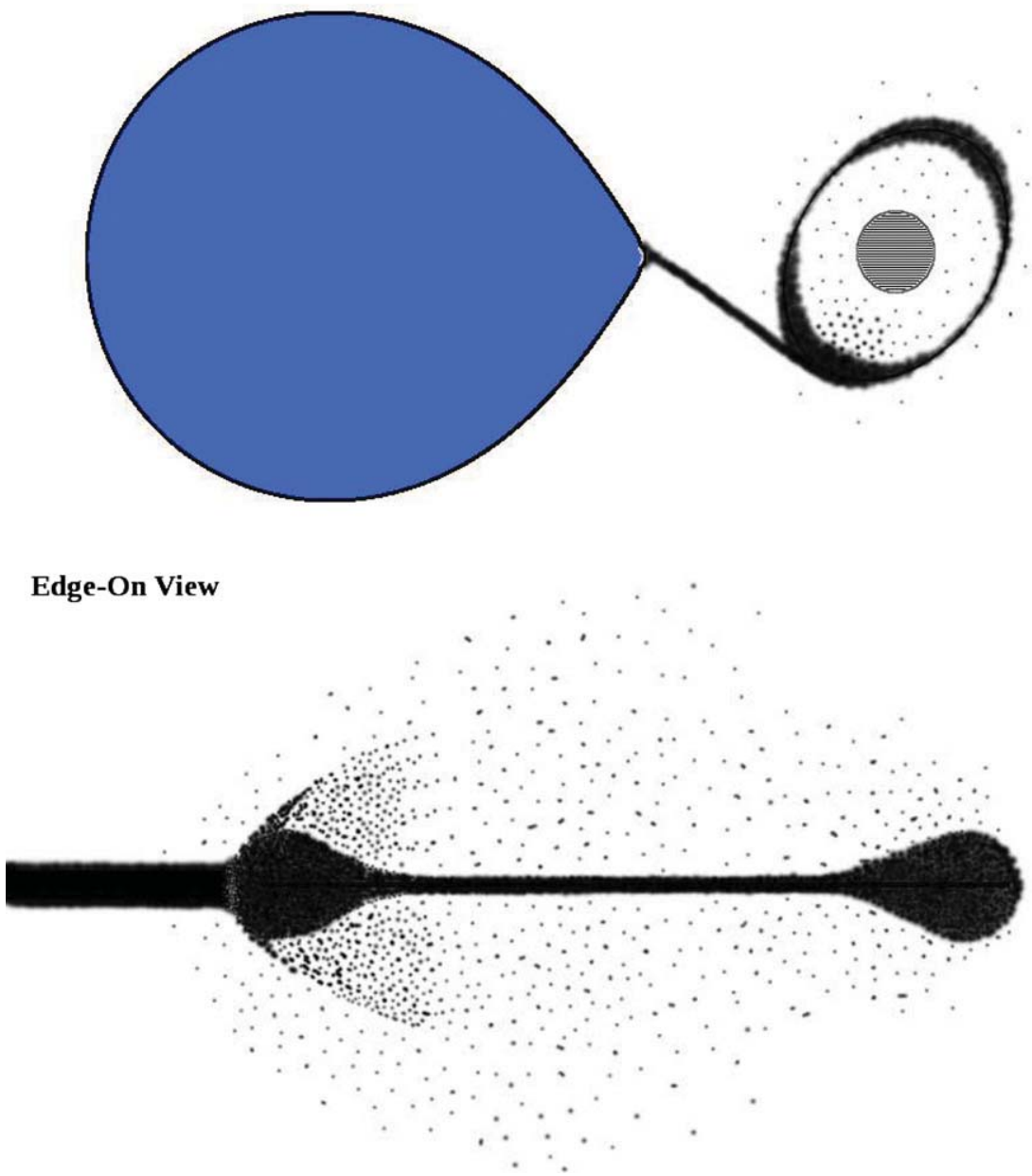

Figure 10. Idealized depiction of a possible accretion geometry for NGC 5408 X-1. Top panel: a face-on (top) view of the binary system. The optical star (possibly a B0I supergiant; Grisé et al. 2012) is shown in blue (left), while the eccentric accretion disk with two obscuring regions is shown on the right-hand side. The X-ray source is indicated by a circular region at the center of the disk. Bottom panel: an edge-on view of the accretion disk. The accretion stream from the companion approaches from the left-hand side. Again the two bulges are shown. The "clumps," produced due to the splashing of the accretion stream at the edge of the disk, are also shown (scattered black points). See also Figure 5 of Armitage \& Livio (1996) and the bottom panel of Figure 7 of Armitage \& Livio (1998).

high inclination X-ray binaries (see the right panels of Figure 6 of Armitage \& Livio 1996). Similar double-peaked modulation profiles have also been observed from neutron star LMXBs including X 1916-053 (Smale et al. 1988; Homer et al. 2001), X 0748-676 (Parmar \& White 1988), and 4U 1822-37 (White \& Holt 1982). Again, in those cases the presence of a secondary bulge, presumably due to the tidal effects of the companion, has been suggested. In this context the nature of the accretor (neutron star or black hole) is largely irrelevant; it is simply the tidal effects on the disk that are important in producing a secondary bulge.

Further simulations by Armitage \& Livio (1998) show that the fate of the material in the accretion stream after it impacts the disk depends on the cooling efficiency within the shock-heated gas created by the impact. If the cooling is efficient, which is generally the case for low-mass accretion rates $\left(\lesssim 10^{-9} M_{\odot} \mathrm{yr}^{-1}\right)$ (see Armitage \& Livio 1998 for details), the material continues to freely overflow above and below the accretion disk. This ballistic stream will reimpact the disk near the point of its closest approach to the compact source where it forms an obscuring bulge (See also Frank et al. 1987; Lubow 1989). However, if the cooling is inefficient which is thought to be the case for high-mass accretion rates, the stream-disk interaction leads to a flow that is better described as an "explosion" at the point of the impact, and leads to a more vertically extended distribution of scattered material (clumps: see the bottom panel of Figure 7 of Armitage \& Livio 1998). Such clumps could account for the sharp dips observed in NGC 5408 X-1. Given its high luminosity and hence high inferred accretion rate (average $\mathrm{X}$-ray luminosity of $\approx 10^{40} \mathrm{erg} \mathrm{s}^{-1}$ ) it is plausible that the cooling at the stream-disk impact site is inefficient. Therefore, one would expect a distribution of splashed material (clumps) around the primary bulge. This is in reasonable agreement with the modulation profile of NGC 5408 X-1 where the sharp dips occur predominantly at one of the minima which is consistent with the phase of the stream-disk impact site (primary bulge).

In summary, the observed modulation profile of NGC 5408 $\mathrm{X}-1$ appears consistent with the predicted variations from Roche-lobe accreting binaries with low-mass ratios, $q=$ $M_{\text {donor }} / M_{\text {accretor }}$ (Armitage \& Livio 1996, 1998). The smooth component is produced by absorption/obscuration by the two spatially distinct bulges along the outer rim of the accretion disk while the sharp dips are possibly produced due to absorption by clumps of material produced by the stream-disk 
impact. A schematic depiction of a possible source geometry for NGC 5408 X-1 based on this scenario is shown in Figure 10. The top panel shows the distribution of material in the binary viewed from above (face on) while the bottom panel shows the accretion disk viewed edge-on.

The sharp dips seen in NGC 5408 X-1 appear similar to those observed in the black hole LMXB GRO J1655-40 (Kuulkers et al. 2000), however, GRO J1655-40 does not show a smooth periodic modulation as in NGC $5408 \mathrm{X}-1$. This may be due to the inclination of GRO J1655-40 $\left(i=70.2 \pm 2^{\circ}\right.$; Greene et al. 2001), as simulations show that the bulge material has a limited extent above the disk. However, stream material splashing on to the edge of the accretion disk is able to reach higher above the plane of the accretion disk (higher than the bulges) so that in principle sharp dips can still occur away from the plane of the accretion disk (Armitage \& Livio 1998). While the morphology of the sharp X-ray dips in NGC 5408 X-1 somewhat resembles partial eclipse profiles, we note that this is unlikely to be the case due to the fact that the sharp dips are not strictly periodic. That is, the large variance on the dip period (see Figure 3) strongly indicates that we are not seeing eclipses.

If these two arguments are correct then a rough constraint on the inclination angle can be derived, as the inclination angle has to be less than some upper limit so that eclipses are not seen but must be large enough so that the absorbing/obscuring effects of the disk bulges are not completely eliminated. Assuming the donor star fills its Roche lobe, an upper limit on the inclination angle (such that eclipses are not seen) can be derived that depends on the orbital period and the mass ratio of the binary (see Eggleton 1983; Rappaport \& Joss 1986 for the appropriate formulae). For an orbital period of 243 days with a B0I donor of $\approx 10 M_{\odot}$, the upper limits on the inclination are $75^{\circ}$ and $85^{\circ}$ assuming the compact source is a massive stellar-mass black hole (mass $\approx 50 M_{\odot}$; Middleton et al. 2011) or an intermediate-mass black hole (mass $\approx 1000 M_{\odot}$; Strohmayer \& Mushotzky 2009), respectively. If we further assume that the absence of a smooth modulation in GRO J1655-40 provides a rough lower limit of $70^{\circ}$, we obtain an inclination angle for NGC $5408 \mathrm{X}-1$ in the range from $70^{\circ}-85^{\circ}$. While this is based on a number of assumptions, we note that it is broadly consistent with inclination angles inferred for other dipping binaries (see Ritter \& Kolb 2003).

Finally, we note that while the sharp dips seen in NGC 5408 $\mathrm{X}-1$ are similar to the type-B dips observed in Cygnus X-1 (see Section 1 for details on the sharp dips from Cygnus X-1), the high X-ray luminosity and thus high inferred mass accretion rate is much easier to accommodate via Roche overflow than wind-fed accretion.

\subsection{Alternative Scenarios}

Alternatively, it could be that the observed X-ray modulations from NGC 5408 X-1 are not due to the orbital motion of the X-ray source in a binary (Foster et al. 2010). X-ray modulations on "super-orbital" periods (periods longer than the binary orbital period) of the order of a few days to a few hundred days have been reported from accreting X-ray binaries (e.g., Wen et al. 2006; Sood et al. 2007). In a sub-sample of these sources consisting of Her X-1 ( $P_{\text {Superorbit }}=35$ days), SS 433 $\left(P_{\text {Superorbit }}=164\right.$ days $)$, LMC X-4 $\left(P_{\text {Superorbit }}=30.4\right.$ days $)$, and SMC X-1 ( $P_{\text {Superorbit }} \approx 55$ days), it is likely that the observed $\mathrm{X}$-ray modulation is due to the periodic obscuration of the central X-ray source by a tilted, precessing accretion disk. The basic idea here is that the accretion disk is tilted or warped with respect to the orbital plane of the binary. The tidal effects of the companion star then force the accretion disk to precess about an axis normal to the binary orbit. As the tilted disk precesses, the effective area of the disk obscuring the central $\mathrm{X}$-ray source can vary, producing modulations at or near the precession period. Furthermore, the radiation force from the $\mathrm{X}$-ray source can warp the outer regions of the accretion disk (Ogilvie \& Dubus 2001; Ogilvie 2002) leading to two spatially distinct density enhancements. This might possibly account for the twin-peaked X-ray modulation curve of NGC 5408 X-1. In summary, the theory of tilted, warped accretion disks may be adaptable to the present scenario to perhaps explain the observed properties.

\section{SUMMARY}

We have presented results from long-term X-ray $(0.3-8.0 \mathrm{keV})$ monitoring of the ULX NGC 5408 X-1 using Swift/XRT. Our primary results are: (1) the discovery of sharp, energyindependent dips (a total of five dip epochs) in the X-ray intensity that recur roughly every 243 days and (2) the detection of a smooth, quasi-sinusoidal modulation of the X-rays which appears to weaken during the second half of the monitoring program. We interpret these findings in the context of orbital motion in a Roch-lobe overflow binary with a period comparable to the dip-recurrence period ( $243 \pm 23$ days; see Figure 10), however, it is also possible that a precessing accretion disk (a "super-orbital" phenomenon) can cause a similar X-ray modulation. Further X-ray monitoring of NGC 5408 X-1 with Swift is warranted to more firmly establish that the X-ray dips are indeed associated with the orbital period of the binary.

We acknowledge support through NASA's Swift Guest Investigator program, and we thank the referee for detailed comments which helped us improve this paper.

\section{REFERENCES}

Armitage, P. J., \& Livio, M. 1996, ApJ, 470, 1024

Armitage, P. J., \& Livio, M. 1998, ApJ, 493, 898

Arnaud, K. A. 1996, in ASP Conf. Ser. 101, Astronomical Data Analysis Software and Systems V, ed. G. H. Jacoby \& J. Barnes (San Francisco, CA: ASP), 17

Bałucińska-Church, M., Church, M. J., Charles, P. A., et al. 2000, MNRAS, 311,861

Barnard, R., Balucińska-Church, M., Smale, A. P., \& Church, M. J. 2001, A\&A, 380, 494

Begelman, M. C. 2002, ApJL, 568, L97

Bisikalo, D. V., Kaigorodov, P. V., Boyarchuk, A. A., \& Kuznetsov, O. A. 2005, ARep, 49, 701

Blondin, J. M., Stevens, I. R., \& Kallman, T. R. 1991, ApJ, 371, 684

Boirin, L., Parmar, A. N., Barret, D., Paltani, S., \& Grindlay, J. E. 2004, A\&A, 418, 1061

Church, M. J. 2001, AdSpR, 28, 323

Church, M. J., Dotani, T., Balucinska-Church, M., et al. 1997, ApJ, 491, 38

Church, M. J., Parmar, A. N., Balucinska-Church, M., et al. 1998, A\&A, 338, 556

Colbert, E. J. M., \& Mushotzky, R. F. 1999, ApJ, 519, 89

Courvoisier, T. J.-L., Parmar, A. N., Peacock, A., \& Pakull, M. 1986, ApJ, 309,265

Davies, M. B., Miller, M. C., \& Bellovary, J. M. 2011, ApJL, 740, L42

Dheeraj, P. R., \& Strohmayer, T. E. 2012, ApJ, 753, 139

Eggleton, P. P. 1983, ApJ, 268, 368

Feng, Y. X., \& Cui, W. 2002, ApJ, 564, 953

Foster, D. L., Charles, P. A., \& Holley-Bockelmann, K. 2010, ApJ, 725, 2480

Frank, J., King, A., \& Raine, D. J. (ed.) 2002, Accretion Power in Astrophysics (Cambridge: Cambridge Univ. Press), 398

Frank, J., King, A. R., \& Lasota, J.-P. 1987, A\&A, 178, 137

Greene, J., Bailyn, C. D., \& Orosz, J. A. 2001, ApJ, 554, 1290 
Grisé, F., Kaaret, P., Corbel, S., et al. 2012, ApJ, 745, 123

Han, X., An, T., Wang, J.-Y., et al. 2012, RAA, 12, 1597

Homer, L., Charles, P. A., Hakala, P., et al. 2001, MNRAS, 322, 827

Horne, J. H., \& Baliunas, S. L. 1986, ApJ, 302, 757

Kaaret, P., \& Feng, H. 2007, ApJ, 669, 106

King, A. R., Davies, M. B., Ward, M. J., Fabbiano, G., \& Elvis, M. 2001, ApJL, 552, L109

Körding, E., Falcke, H., \& Markoff, S. 2002, A\&A, 382, L13

Kuulkers, E., in't Zand, J. J. M., Cornelisse, R., et al. 2000, A\&A, 358, 993

Kuulkers, E., Kouveliotou, C., Belloni, T., et al. 2012, arXiv:1204.5840

Kuulkers, E., Wijnands, R., Belloni, T., et al. 1998, ApJ, 494, 753

Lubow, S. H. 1989, ApJ, 340, 1064

Magorrian, J., Tremaine, S., Richstone, D., et al. 1998, AJ, 115, 2285

Mason, K. O. 1986, in The Physics of Accretion onto Compact Objects, ed. K. P. Mason, M. G. Watson, \& N. E. White (Lecture Notes in Physics, Vol. 266; Berlin: Springer), 29

McClintock, J. E., \& Remillard, R. A. 2006, in Compact Stellar X-Ray Sources, ed. W. Lewin \& M. van der Klis (Cambridge Astrophysics Ser. 39; Cambridge: Cambridge Univ. Press), 157

Middleton, M. J., Roberts, T. P., Done, C., \& Jackson, F. E. 2011, MNRAS, 411,644

Ogilvie, G. I. 2002, MNRAS, 330, 937

Ogilvie, G. I., \& Dubus, G. 2001, MNRAS, 320, 485
Parmar, A. N., \& White, N. E. 1988, MmSAI, 59, 147

Parmar, A. N., White, N. E., Giommi, P., \& Gottwald, M. 1986, ApJ, 308, 199

Rappaport, S. A., \& Joss, P. C. 1983, Accretion-Driven Stellar X-ray Sources, 13

Ritter, H., \& Kolb, U. 2003, A\&A, 404, 301

Scargle, J. D. 1982, ApJ, 263, 835

Smale, A. P., Church, M. J., \& Bałucińska-Church, M. 2002, ApJ, 581, 1286

Smale, A. P., Mason, K. O., White, N. E., \& Gottwald, M. 1988, MNRAS, 232, 647

Sood, R., Farrell, S., O’Neill, P., \& Dieters, S. 2007, AdSpR, 40, 1528

Soria, R., Motch, C., Read, A. M., \& Stevens, I. R. 2004, A\&A, 423, 955

Strohmayer, T. E. 2009, ApJL, 706, L210

Strohmayer, T. E., \& Mushotzky, R. F. 2009, ApJ, 703, 1386

Strohmayer, T. E., Mushotzky, R. F., Winter, L., et al. 2007, ApJ, 660, 580

Swartz, D. A., Ghosh, K. K., Tennant, A. F., \& Wu, K. 2004, ApJS, 154, 519

Volonteri, M., Haardt, F., \& Madau, P. 2003, ApJ, 582, 559

Wen, L., Levine, A. M., Corbet, R. H. D., \& Bradt, H. V. 2006, ApJS, 163,372

White, N. E., \& Holt, S. S. 1982, ApJ, 257, 318

White, N. E., Nagase, F., \& Parmar, A. N. 1995, in X-ray Binaries, ed. W. H. G. Lewin, J. van Paradijs, \& E. P. J. van den Heuvel (Cambridge: Cambridge Univ. Press), 1 